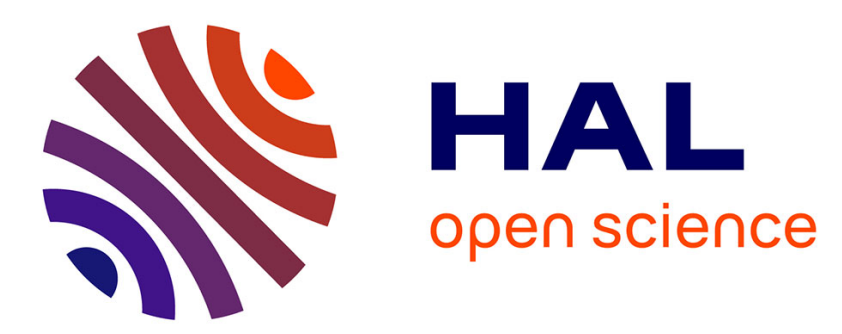

\title{
Evidence for different in vitro oligomerization behaviors of synthetic hIAPP obtained from different sources
}

Corentin Berardet, Julia Kaffy, Frédéric Halgand, Guillaume van Der Rest, Sandrine Ongeri, Myriam Taverna

\section{- To cite this version:}

Corentin Berardet, Julia Kaffy, Frédéric Halgand, Guillaume van Der Rest, Sandrine Ongeri, et al.. Evidence for different in vitro oligomerization behaviors of synthetic hIAPP obtained from different sources. Analytical and Bioanalytical Chemistry, 2020, 412 (13), pp.3103-3111. 10.1007/s00216-02002560-5 . hal-03011853

\section{HAL Id: hal-03011853 \\ https://hal.science/hal-03011853}

Submitted on 2 Dec 2020

HAL is a multi-disciplinary open access archive for the deposit and dissemination of scientific research documents, whether they are published or not. The documents may come from teaching and research institutions in France or abroad, or from public or private research centers.
L'archive ouverte pluridisciplinaire HAL, est destinée au dépôt et à la diffusion de documents scientifiques de niveau recherche, publiés ou non, émanant des établissements d'enseignement et de recherche français ou étrangers, des laboratoires publics ou privés. 


\title{
Evidence for different in vitro oligomerization behaviors of synthetic hIAPP obtained from different sources
}

\author{
Corentin Berardet ${ }^{1,2} \cdot$ Julia Kaffy $^{2} \cdot$ Frédéric Halgand $^{3} \cdot$ Guillaume Van der Rest $^{3} \cdot$ Sandrine Ongeri $^{2}$. \\ Myriam Taverna ${ }^{1,4}$
}

Received: 18 November 2019 / Revised: 7 February 2020 / Accepted: 28 February 2020

(C) Springer-Verlag GmbH Germany, part of Springer Nature 2020

\begin{abstract}
Type 2 diabetes is characterized by the aggregation of human islet amyloid polypeptide (hIAPP), from monomer to amyloid deposits that are made of insoluble fibrils. Discrepancies concerning the nature of formed species or oligomerization kinetics among reported in vitro studies on hIAPP aggregation process have been highlighted. In this work, we investigated if the sample itself could be at the origin of those observed differences. To this aim, four hIAPP samples obtained from three different sources or suppliers have been analyzed and compared by ThT fluorescence spectroscopy and by two recently developed techniques, capillary electrophoresis (CE), and ESI-IMS-QToF-MS. Lots provided by the same supplier were shown to be very similar whatever the analytical technique used to characterize them. In contrast, several critical differences could be pointed out for hIAPP provided by different suppliers. We demonstrated that in several samples, some oligomerized peptides (e.g., dimer) were already present upon reception. Purity was also different, and the proneness of the peptide solution to form fibrils in vitro within $24 \mathrm{~h}$ could vary considerably from one sample source to another but not from lot to lot of the same source. All those results demonstrate that the initial state of conformation, oligomerization, and quality of the hIAPP can greatly impact the aggregation kinetics, and thus the information provided by these in vitro tests. Finally, a careful selection of the peptide batch and source is mandatory to perform relevant in vitro studies on hIAPP oligomerization and to screen new molecules modulating this pathological process.
\end{abstract}

Keywords Human amylin · Capillary electrophoresis · Ion mobility mass spectrometry - ThT fluorescence spectroscopy · Aggregation · Amyloid · Type 2 diabetes

\section{Introduction}

Affecting more than 400 million people in the world, type 2 diabetes (T2D) is now considered a major public health issue [1]. Treatments are only non-etiological, considering that the

Myriam Taverna

myriam.taverna@universite-paris-saclay.fr

1 CNRS, Institut Galien Paris Sud, Université Paris-Saclay, 92296 Châtenay-Malabry, France

2 CNRS, BioCIS, FLUOPEPIT, Université Paris-Saclay, 92290 Châtenay-Malabry, France

3 Institut de Chimie Physique, UMR 8000, Réactivité des Ions, Spectrométrie de Masse, Analyse et Spectroscopies (RISMAS), CNRS, University Paris-Sud, University Paris-Saclay, Rue Henri Becquerel, Bâtiment 201 P2, 91405 Orsay, France

4 Institut Universitaire de France, Paris, France cause of this disease is not elucidated yet [2]. In $96 \%$ of diabetic patients, extracellular amyloid deposits composed of amyloid fibrils of islet amyloid polypeptide (hIAPP) are found in the pancreas [3]. hIAPP (or amylin) is produced by proteolytic cleavage of the pro-islet amyloid polypeptide and is cosecreted with insulin in a 1:100 molar ratio [3]. During the pathological amyloid cascade, an aggregation process which ultimately leads to the formation of amyloid plaques, morphologically distinct species of different sizes and solubilities are formed such as oligomers, protofibrils, and fibrils [2]. In the last decade, a new hypothesis has emerged concerning the role of hIAPP in T2D, and potential toxicity of the related species like oligomers $[2,4-6]$. This hypothesis tends to consider that small oligomers are mostly responsible for the toxicity towards $\beta$-cells.

Stable and insoluble hIAPP fibrils have been the most studied species up to now, using several analytical techniques like TEM [7, 8], Thioflavin T (ThT) fluorescence spectroscopy 
[9], and DLS [10, 11]. Little work has been devoted to the study, at a molecular level, of hIAPP oligomerization from the earliest stages of the process, while it would give insights on the parameters triggering or stopping this pathological process. In vitro studies investigating this pathological process have been based mostly on fluorescence spectrophotometry using ThT as a $\beta$-sheet probe [12-19], size exclusion liquid chromatography [18], SDS-PAGE [18, 20], NMR [8, 21, 22], or IMS-MS [15, 19, 23-26]. However, these studies have provided different (if not conflicting) results, in terms of observed species (size and conformations of hIAPP) and of kinetics rate of the oligomerization process. These discrepancies could be attributed to the differences in the techniques employed. However, several studies supported by the same techniques have also come to different conclusions, showing that there are other sources of variability.

Marek and co-workers have shown in 2012 that the ionic strength and the $\mathrm{pH}$ of the incubation solution significantly affected hIAPP fibrillization rate as monitored by ThT fluorescence spectroscopy [27]. At low $\mathrm{pH}$, they pointed out that even the nature of the buffer anion had an impact on hIAPP oligomerization. In 2017, Sebastiao et al. have demonstrated that the measurement frequency in ThT fluorescence spectroscopy experiments can even modify hIAPP fibrillization kinetics, indicating that agitation and slight displacements of a microplate inside the spectrophotometer were sufficient to significantly affect it [14]. On the other hand, Suzuki and coworkers have shown that the presence of ThT in the incubation medium did not modify the kinetics rate [28]. All these results strongly indicate that analytical parameters (measurement intervals, temperature ...) can be a variability source, as well as incubation conditions (buffers nature, concentration, $\mathrm{pH} . .$.$) .$

Besides, Suvorina and co-workers have demonstrated the polymorphism that could arise for $A \beta_{1-42}$ fibrils purchased from different suppliers, although incubated in the same medium. Whereas the variability has not been demonstrated on early formed species, this study showed that the peptide source can have a strong impact on its aggregation behavior [29]. In 2008, our group already demonstrated differences in the $A \beta_{1-42}$ samples provided by different suppliers and already hypothesized that impurities in the peptide preparations could have a significant impact on the spontaneous aggregation of peptides as they might function as seeds for aggregation [30]. It appeared to us then that variability may also arise from the production method of the peptide and thus from the state, purity, and quality of the different hIAPP batches employed for these studies.

Considering that no work has been reported to date about the variability induced by hIAPP provenance, we have deeply investigated whether its source had an influence on the in vitro oligomerization and fibrillization behavior of this peptide. To achieve this goal, we combined different techniques (CE-UV, ESI-IMS-QToF-MS, ThT spectroscopy) to provide a comprehensive and new way to characterize in-depth hIAPP obtained from three different suppliers. Two were commercially available, and one was produced by a custom peptide synthesis platform. We also investigated the variability of different batches of the same supplier. We characterized both fresh peptide samples and stored 1 day at room temperature to get insight on their aggregation behavior.

\section{Materials and methods}

\section{Chemicals}

Four batches of hIAPPs (TFA salt) were purchased from 3 different suppliers: Bachem, supplier A (Bubendorf, Switzerland); Sigma, supplier B (St. Louis, MO, USA); and custom peptide synthesis platform, supplier C (University of Cergy-Pontoise, Cergy-Pontoise, France) called batch A1, A2, B, and C, respectively. Details of attributes available in CoAs for each batch have been reported in Table 1. Sodium hydroxide was obtained from VWR (Fontenay-sous-Bois, France) and sodium chloride from Euromedex (Strasbourg, France). Ammonium acetate, DMSO, hexafluoroisopropanol (HFIP), methanol, hexadimethrine bromide "polybrene" (PB, MW 4000-6000 g/mol), and Thioflavin T were obtained from Sigma (St. Louis, MO, USA). All buffers were prepared in

Table 1 Details of attributes for each batch used in the study

\begin{tabular}{|c|c|c|c|c|}
\hline Test & $\begin{array}{l}\text { Bachem (A1) } \\
3016548\end{array}$ & $\begin{array}{l}\text { Bachem (A2) } \\
7001244\end{array}$ & $\begin{array}{l}\text { Sigma (B) } \\
\text { SLBF1570V }\end{array}$ & Cergy (C) \\
\hline Appearance & White powder & White powder & White powder & White powder \\
\hline Solubility & $\begin{array}{l}\text { Clear and colorless solution } \\
\text { in DMF at } 1 \mathrm{mg} / \mathrm{mL}\end{array}$ & $\begin{array}{c}\text { Clear and colorless solution in } \\
5 \% \text { acetic acid at } 1 \mathrm{mg} / \mathrm{mL}\end{array}$ & $\begin{array}{l}\text { Clear and colorless solution } \\
\text { in } \mathrm{H}_{2} \mathrm{O} \text { at } 1 \mathrm{mg} / \mathrm{mL}\end{array}$ & $\mathrm{NA}^{* *}$ \\
\hline Peptide content by AAA* $(\%)$ & 84.6 & 84.9 & 88 & $\mathrm{NA}^{* *}$ \\
\hline Purity (\%) & 95.1 & $\geq 96$ & 97 & $90-95$ \\
\hline
\end{tabular}

*Amino acid analysis

**Not analyzed 
deionized water. Deionized water was prepared with a Direct$\mathrm{Q}^{\circledR} 3$ Water Purification System form Millipore (Milford, USA).

\section{Sample preparation}

All hIAPP samples were dissolved upon reception in $100 \%$ HFIP and incubated for $1 \mathrm{~h}$ at room temperature. After aliquoting them, samples were dried at $40{ }^{\circ} \mathrm{C}$ under vacuum and then stored at $-20^{\circ} \mathrm{C}$. Dried peptides were reconstituted in $50 \mathrm{mM}$ ammonium acetate buffer, $\mathrm{pH} 3.7$, at a concentration of $100 \mu \mathrm{M}$ at room temperature just before performing the kinetics experiments. The same sample preparation was used for CE-UV and ESI-QToF-MS experiments. For Thioflavin T experiments only, the peptide was reconstituted in $100 \%$ DMSO at $200 \mu \mathrm{M}$. For ESI-QToF-MS experiments, the stock solutions were diluted to $5 \mu \mathrm{M}$ for batch $\mathrm{A}$ and $10 \mu \mathrm{M}$ for batches B and $\mathrm{C}$.

\section{Capillary electrophoresis}

$\mathrm{CE}$ experiments were carried out with a P/ACE MDQ (SCIEX, Brea, CA, USA) equipped with a UV detection at $200 \mathrm{~nm}$. Bare fused silica capillaries $(60 \mathrm{~cm}$ total length $\times$ $50 \mu \mathrm{m}$ id) were purchased from Polymicro Technologies (Phoenix, AZ, USA). The polybrene (PB) coating procedure was previously described [31]. Briefly, the silica capillary was preconditioned with $\mathrm{MeOH}, 1 \mathrm{M} \mathrm{NaOH} 1 \mathrm{M}$, and $0.1 \mathrm{M}$ $\mathrm{NaOH} 0.1 \mathrm{M}$ (20 psi for $15 \mathrm{~min})$. The capillary was then coated with the $0.2 \%(\mathrm{w} / \mathrm{v})$ polybrene solution for $15 \mathrm{~min}$ at 20 psi. Capillary was finally equilibrated with the running buffer prior to the analysis.

\section{ThT fluorescence spectroscopy}

ThT spectroscopy experiments were carried out with a microplate reader (FLUOstar Optima, BMG LABTECH, Ortenberg, Germany) equipped with 440/485 excitation/emission filters. Ninety-six-well plates were used. Buffer was composed of $10 \mathrm{mM}$ Tris/ $\mathrm{HCl}, 100 \mathrm{mM} \mathrm{NaCl}$, and $10 \mu \mathrm{M}$ ThT and was adjusted to $\mathrm{pH}$ 7.4. Each well was filled with $195 \mu \mathrm{L}$ of buffer and $5 \mu \mathrm{L}$ of hIAPP solution $(5 \mu \mathrm{M}$ final concentration).

\section{ESI-QToF-MS experiments}

Mass spectrometry experiments were performed on a Synapt G2-S ${ }^{\mathrm{i}}$ HDMS Q-ToF instrument (Waters, Manchester, UK) equipped with a traveling wave ion mobility cell. In this device, the electric field which is applied in a small region, moves along the tube to transport ions to the detector, then allowing ion fractionation according to their size, charge state, shape, and collisional cross section [32]. All samples were

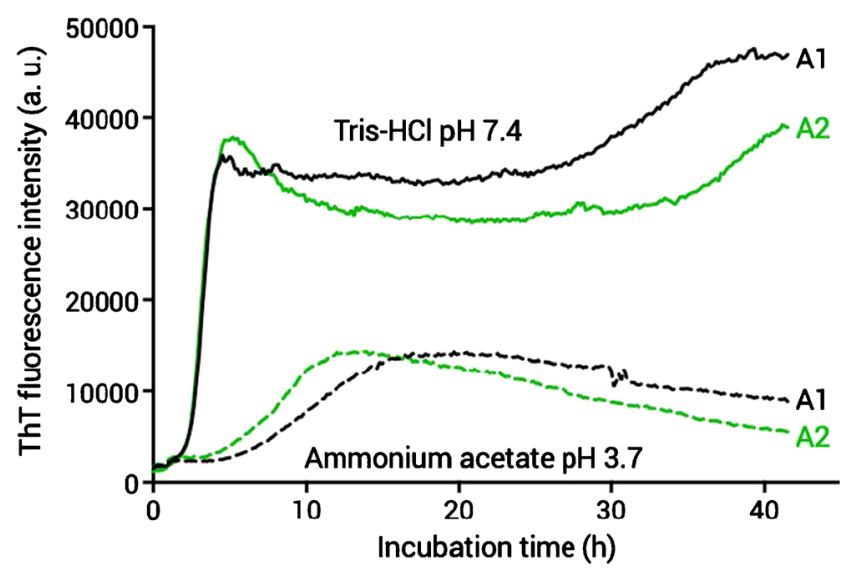

Fig. 1 ThT fluorescence spectroscopy monitoring over time of aggregation of hIAPP from batches A1 and A2 (5 $\mu \mathrm{M}$ in DMSO) and incubated in two different buffers: $10 \mathrm{mM}$ Tris- $\mathrm{HCl} / 100 \mathrm{mM} \mathrm{NaCl}$ at $\mathrm{pH} 7.4+10 \mu \mathrm{M}$ ThT (plain lines) and $50 \mathrm{mM}$ ammonium acetate at pH $3.7+10 \mu \mathrm{M}$ ThT (dotted lines); $\lambda_{\text {exc }} 440 \mathrm{~nm}, \lambda_{\mathrm{em}} 485 \mathrm{~nm}$

infused to the ion source at $5 \mu \mathrm{L} / \mathrm{min}$ flow rate. Acquisition of mass spectra was carried out over the 500 to $5000 \mathrm{~m} / \mathrm{z}$ mass range, and $\mathrm{m} / \mathrm{z}$ calibration was performed using sodium trifluoroacetate leading to a typical error below $2 \mathrm{ppm}$. In order to preserve, as much as possible, oligomeric complexes in the gas phase, we used "native" conditions as follows: positive ion mode was chosen regarding hIAPP pI $(\sim 8.6)$ and acidic analysis conditions identical to those used for CE experiments (ammonium acetate $50 \mathrm{mM}, \mathrm{pH}$ 3.7). Experimental parameters set on the instrument were the following: capillary voltage $2.4 \mathrm{kV}$, sampling cone $70 \mathrm{~V}$, source offset $70 \mathrm{~V}$, source temperature $40{ }^{\circ} \mathrm{C}$, desolvation gas flow $500 \mathrm{~L} / \mathrm{h}$. For ion mobility experiments, instrumental parameters were as follows: $\mathrm{N}_{2}$ as the drift gas, trap gas flow $2 \mathrm{~mL} / \mathrm{min}$, IMS gas flow $90 \mathrm{~mL} / \mathrm{min}$, trap wave velocity $125 \mathrm{~m} / \mathrm{s}$ (300 for batch $\mathrm{C}$ ), trap wave height $6 \mathrm{~V}$ (4 for supplier C), He cell gas flow $180 \mathrm{~mL} / \mathrm{min}$, IMS wave velocity $550 \mathrm{~m} / \mathrm{s}$ (950 for batch C), IMS wave height $25 \mathrm{~V}$ (40 for batch C), transfer wave velocity

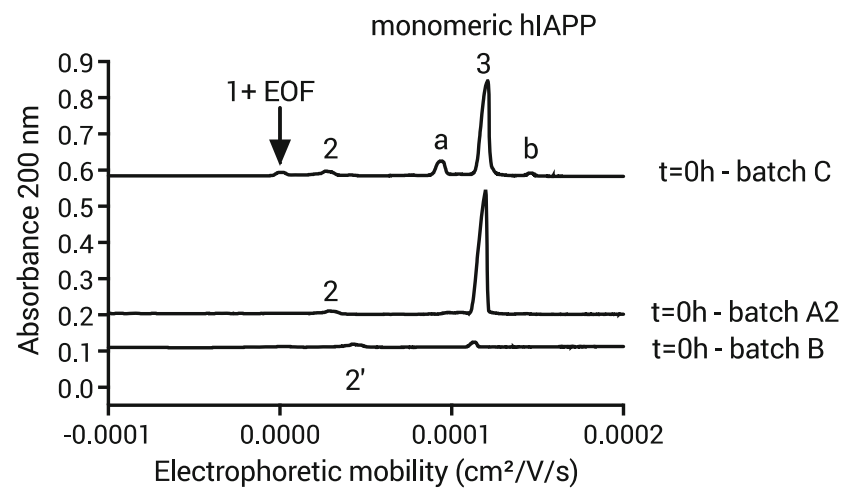

Fig. 2 Electropherograms of hIAPP obtained for batches A2, B, and C, using polybrene-coated capillaries $(60 \mathrm{~cm} \times 50 \mu \mathrm{m})$ with a BGE composed of $50 \mathrm{mM}$ ammonium acetate at $\mathrm{pH}$ 3.7. Other conditions: applied voltage $-25 \mathrm{kV}$, detection $200 \mathrm{~nm}$. For each batch, hIAPP was reconstituted at $100 \mu \mathrm{M}$ in the $\mathrm{BGE}$ and analyzed immediately 
$63 \mathrm{~m} / \mathrm{s}$ (110 for batch C), and transfer wave height $=4 \mathrm{~V}$. CCS have been calibrated with ubiquitin. For the transfer wave velocity, $63 \mathrm{~m} / \mathrm{s}$ was initially chosen to work on a $\mathrm{m} / \mathrm{z}$ range up to $14,000 \mathrm{Da}$. When analyzing batch $\mathrm{C}$, parameters were slightly optimized, and this range was reduced to $\mathrm{m} / \mathrm{z} 5000$ which corresponds to a wave velocity of $110 \mathrm{~m} / \mathrm{s}$. This optimization did not affect conclusions made on differences between batches.

\section{Results and discussion}

\section{Dissolution medium and incubation buffer}

We first studied the impact of the dissolution medium and sample preparation on the hIAPP state upon sample reception. Different protocols of dissolution were tested on one commercial hIAPP sample (batch A1). Prior to the reconstitution of samples in the incubation medium, all commercial and dried peptides were cleaned up from potential aggregated species using an HFIP treatment, followed by its evaporation under vacuum and storage at $-20{ }^{\circ} \mathrm{C}$. Two different incubation times for the HFIP treatment were first tested: $1 \mathrm{~h}$ and one night. ThT fluorescence spectroscopy, which allows the monitoring of $\beta$-sheet-rich structures formation over time, was then used to evaluate the efficiency of this treatment and the impact of the incubation time. Samples were analyzed immediately after their reconstitution in a $10 \mathrm{mM}$ Tris- $\mathrm{HCl}+$ $100 \mathrm{mM} \mathrm{NaCl}, \mathrm{pH}$ 7.4. The obtained fluorescence intensity curves did not exhibit any difference, neither on the lag phase duration nor on the plateau intensity. We kept the 1-h dissolution/incubation in HFIP for subsequent studies.
As the incubation buffer used to mimic in vitro hIAPP aggregation may have a profound impact on this process, we tested two different media: $10 \mathrm{mM}$ Tris- $\mathrm{HCl}$ containing $100 \mathrm{mM} \mathrm{NaCl}, \mathrm{pH} 7.4$, and $50 \mathrm{mM}$ ammonium acetate, $\mathrm{pH}$ 3.7, by measuring the ThT fluorescence intensity over time (Fig. 1). This experiment was repeated on two different batches A1 and A2, both provided however by the same supplier. We observed that the curves were superimposable whatever the employed medium showing that the two peptide batches were very similar. The two buffers led however to quite different fluorescence curves in terms of lag phase (start of the fluorescence increase), saturation phase (time to reach the plateau), and fluorescence intensity at the plateau. In the Tris- $\mathrm{HCl}$ medium ( $\mathrm{pH}$ 7.4), the lag phase was twice shorter than in the ammonium acetate buffer $(\mathrm{pH} 3.7)$, whereas the intensity of the fluorescence plateau was 3 times higher. This indicates that the ammonium acetate buffer leads to a slower kinetics and forms less or different $\beta$-sheet-rich species of hIAPP. We cannot exclude however that this buffer could contribute itself to decrease partly the fluorescence emission. However, it does not fully explain the differences observed between the two conditions and these results indicate thus that hIAPP behaves differently in those buffers. From these results, the ammonium acetate buffer, having a slower oligomerization rate, has been retained for the further studies on hIAPP oligomerization process by CE and ESI-QToF-MS.

\section{Comparison of hIAPP from three different suppliers}

Just after reconstitution, fresh hIAPP samples from three different suppliers were analyzed by capillary electrophoresis using a method we recently developed [31] and by ESI-
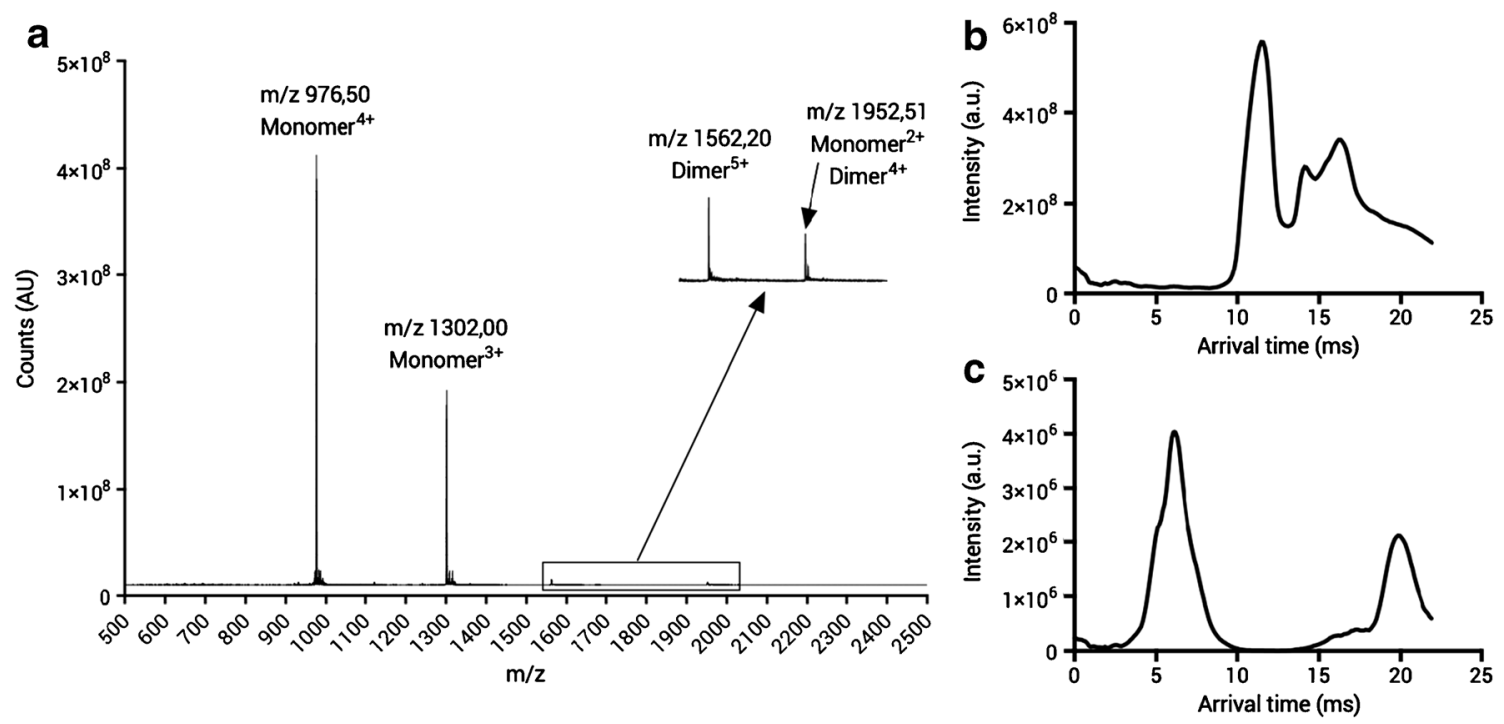

Fig. 3 Mass spectra of hIAPP batch A2 (a) obtained by direct infusion of the hIAPP solution in a Synapt G2-S $\mathrm{i}$ instrument. hIAPP was dissolved in $50 \mathrm{mM}$ ammonium acetate at $\mathrm{pH} 3.7$ at a concentration of $5 \mu \mathrm{M}$. Panel $\mathbf{b}$ shows the global ATD (arrival distribution time) for A2 mass spectrum. Panel $\mathbf{c}$ shows the ATD of monomer ${ }^{2+} /$ dimer $^{4+}$ signal, exhibiting several distributions 
Table 2 Monoisotopic calculated masses for each charge state and each peptide batch, with associated averaged mass for monomer and dimer

\begin{tabular}{llll}
\hline & Batch A2 (Da) & Batch B (Da) & Batch C (Da) \\
\hline Monomer $^{4+}$ & 3901.03 & 3900.90 & 3900.94 \\
Monomer $^{3+}$ & 3901.02 & 3900.90 & 3900.92 \\
Monomer $^{2+}$ & 3901.00 & 3900.89 & 3900.94 \\
Mean (monomer) & 3901.02 & 3900.89 & 3900.93 \\
Dimer $^{4+}$ & 7802.01 & 7801.78 & 7801.88 \\
Dimer $^{5+}$ & 7801.96 & 7801.86 & 7801.84 \\
Mean (dimer) & 7801.98 & 7801.82 & 7801.86 \\
\hline
\end{tabular}

Italic entries show the mean value

QToF-MS to get more insights on their composition and aggregation state.

The CE-UV method uses a PB-coated capillary to avoid hIAPP adsorption onto capillary walls. Capillary electrophoresis profiles exhibited strong differences between batches analyzed under the same conditions and concentrations $(100 \mu \mathrm{M})$ (Fig. 2). Profiles were similar for peptides from batch $\mathrm{A} 2$ or $\mathrm{A} 1$ and $\mathrm{C}$ with one main peak (peak 3) corresponding to the monomeric peptide and two smaller ones (peaks 1 and 2) that were shown to be hIAPP oligomeric species with a MW above $100 \mathrm{kDa}$ [31]. Two additional peaks $\mathrm{a}$ and $\mathrm{b}$ were present in batch $\mathrm{C}$. They correspond either to impurities or to hIAPP-related species like other small oligomers. The peptide from batch B exhibited the same major peak but at a very low intensity (15 times lower). Peaks 1 and 2 were not present in this sample but a new peak with a very low intensity was detected $\left(2^{\prime}\right)$. These results indicate that the peptide in batch $\mathrm{B}$ was already aggregated to a significant proportion. From CE experiments, we can conclude that hIAPP peptides from batches $\mathrm{C}$ and A were very similar, the purity of batch A being however clearly higher. Batch B peptide is probably under an oligomerized or aggregated state too large to have been disaggregated by the HFIP treatment and not enough soluble to appear as an additional peak on the electropherogram.

In order to explain the differences observed between the peptides from different suppliers, ESI-QToF-MS has been used to determine the composition of each peptide batch (Fig. 3). All the calculated and expected masses considered in this section were monoisotopic ones. Results obtained for the three investigated peptides are summarized in Table 2. For all suppliers, four characteristic hIAPP signals were observed and assigned to monomer and dimer. Batch A2 exhibited a peak at $\mathrm{m} / \mathrm{z} 976.25$ $\left(\right.$ monomer $\left.+4: \mathrm{M}^{4+}\right)$, which led to a calculated mass of $3901.03 \mathrm{Da}$, and a second at $\mathrm{m} / \mathrm{z} 1301.34\left(\mathrm{M}^{3+}\right)$ with a calculated mass of $3901.02 \mathrm{Da}$. Both corresponded to the monomer which expected mass is $3900.86 \mathrm{Da}$. Two smaller peaks were observed, corresponding to a dimeric form with an expected mass of $7801.73 \mathrm{Da}\left(\mathrm{m} / \mathrm{z} 1561.39\right.$ for $\mathrm{dimer}^{5+}$ and $\mathrm{m} / \mathrm{z}$ 1951.50 for monome $^{2+} / \mathrm{dimer}^{4+}$ ). For all the three suppliers, calculated masses were similar and consistent with expected masses from the primary sequence.

For batches $\mathrm{B}$ and $\mathrm{C}$, however, an additional peak was observed at $\mathrm{m} / \mathrm{z} 538$ which was likely a contaminant in those peptide samples as its $m / z$ did not correspond to hIAPP. Moreover, the intensity of the hIAPP monomeric signal $(\mathrm{m} / \mathrm{z}$
Fig. 4 a and $\mathbf{b}$ show the global ATD obtained for batches B and $\mathrm{C}$ respectively by direct infusion of the hIAPP solution in a Synapt G2-Si instrument. hIAPP was dissolved in ammonium acetate $50 \mathrm{mM}, \mathrm{pH} 3.7$, at a concentration of $10 \mu \mathrm{M}$ (B and C). No drastic increase of oligomers quantity was observed by increasing the sample concentration (compared with Fig. 3). $\mathbf{c}$ and d show the ATD for monomer ${ }^{2+} /$ dimer $^{4+}$ signal only
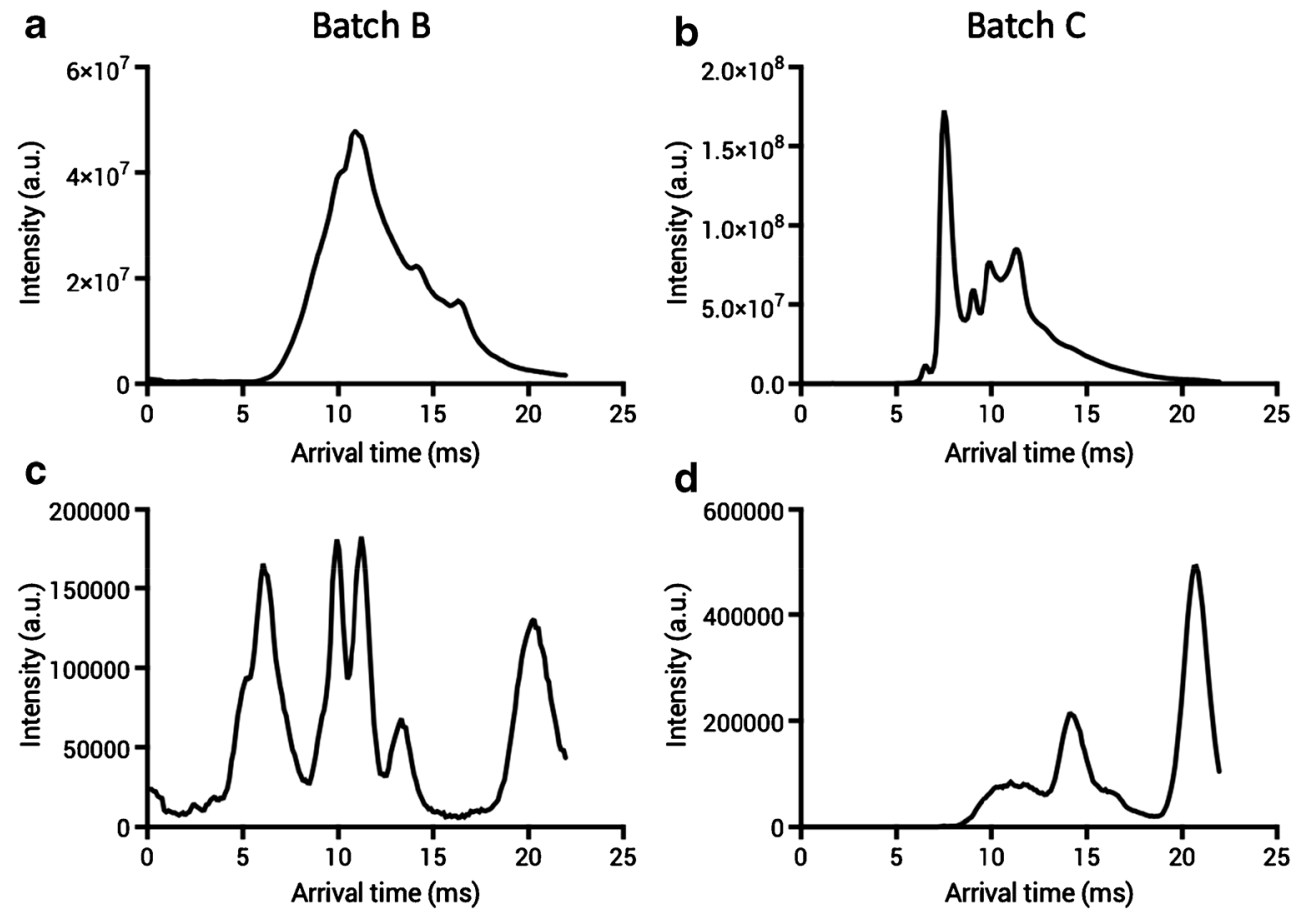
Table 3 Comparison of the aggregation kinetics obtained for the three different batches as monitored by ThT fluorescence assay. For each curve, the lag and saturation phases and the plateau intensity are compared. Values were obtained by applying a sigmoidal fitting on the curve (6 repetitions)

\begin{tabular}{lllr}
\hline Batch & Lag phase (h) & Saturation phase (h) & Fluorescence intensity at the plateau (a.u.) \\
\hline A2 & 2 & 5 & 40,000 \\
B & 5 & 7 & 5000 \\
C & 7 & 12 & 10,000 \\
\hline
\end{tabular}

976) was about 100 times less intense in the peptide from batch $\mathrm{B}$ than in $\mathrm{A} 2$, indicating a much higher monomeric purity in A2. The spectrum from batch $\mathrm{C}$ was more complex. Two new signals at $m / z, 942.47(+4)$ and $1256.29(+3)$ were visible. Regarding those $\mathrm{m} / \mathrm{z}$ and associated charge states, we made the hypothesis that these signals corresponded to the peptide without the C-term tyrosine. Tandem MS was performed selecting $m / z \quad 1256.29$ as a precursor without being able to confirm this hypothesis. Those peaks were considered synthesis impurities. The overall results confirmed the presence of hIAPP in all peptide batches, but certainly with different oligomerization states and purities.

Ion mobility spectroscopy coupled to mass spectrometry allows the identification of species present in each sample and provides also insights on the folding and the conformation of the observed structures. In 2017, Van der Rest's group proposed a method to perform native mass spectrometry in order to preserve complexes and conformers with Prion and other larger proteins $[33,34]$. This method was adapted to the study of hIAPP conformations in each batch (A2, B, and C). A2 batch mass spectrum presented in Fig. 3a exhibited at least 4 characteristic peaks of the peptide. The arrival time distribution (ATD) of the whole spectrum obtained with the IMS feature is presented in Fig. 3b. This figure shows the multiplicity of conformations that were present in the sample. Figure $3 c$ presents only the ATD of monomer ${ }^{2+} /$ dimer $^{4+}$ signal, leading to an easier distribution to focus on. It clearly appears that at least 3 main distributions corresponding to three co-existing conformations of the same species were present here. One should mention that it is likely that signal intensities are not related to their abundance but mostly to their ionization rate. In other words, their intensity does not necessarily reflect their molar ratio.
Fig. 5 CE profiles of hIAPP incubated in $50 \mathrm{mM}$ ammonium acetate at $\mathrm{pH} 3.7$ over $20 \mathrm{~h}$ obtained for the three peptide batches A2 (a), B (b), and C (c). Electropherograms were shifted on the $Y$-axis. CE parameters were the same as in Fig. 2. Peaks 1, 2, and $2^{\prime}$ correspond to hIAPP oligomerized species and peak 3 to hIAPP monomer. Peaks a and $b$ correspond to either impurities or small hIAPP oligomers
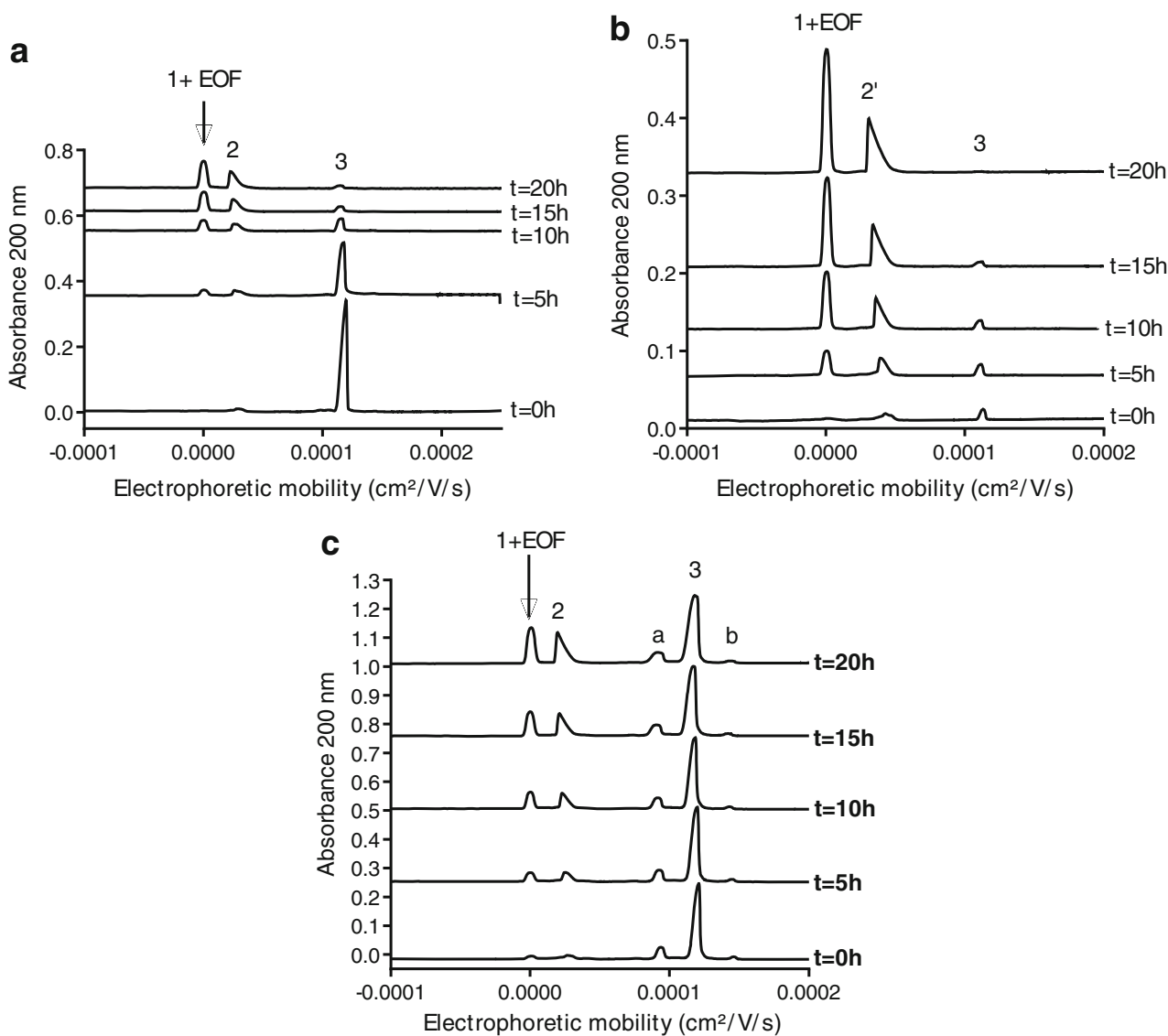
These plots allowed to perform fingerprint comparisons between each batch, highlighting differences in terms of conformation for monomer and dimer on global ATD (Fig. 4a, b) and more specifically on ATD for monomer ${ }^{2+} /$ dimer $^{4+}$ signal (Fig. 4c, d). It appeared that batch B was clearly more complex than $\mathrm{A} 2$ and $\mathrm{C}$, exhibiting much more distributions on both global ATD or/and monomer ${ }^{2+} /$ dimer $^{4+}$ signal. Distribution around $20 \mathrm{~ms}$ was present in all batches. This corresponds probably to the most stable hIAPP conformation. Other distributions were not present in all the studied batches, being probably alternative and less stable conformations. These results demonstrate the high variability existing between all three hIAPP batches, and the need of a careful selection of the peptide source to monitor its oligomerization process.

\section{Impact of the observed differences on the aggregation behavior}

In view of these results, the three suppliers provide hIAPP in different states of conformation and/or aggregation, and with different degrees of purity. We then investigated whether these differences have an impact on the aggregation kinetics. ThT fluorescence spectroscopy data obtained after reconstitution of each peptide lot in a Tris- $\mathrm{HCl}$ medium at $\mathrm{pH} 7.4$ were used to compare the aggregation kinetics of the three peptides under the same conditions of incubation and analysis (Table 2). Some differences in the lag phase, the saturation phase, and the fluorescence intensity at the plateau were clearly pointed out. Peptide from batch A2 oligomerized 3.5 times faster than $\mathrm{C}$, producing also more or tighter $\beta$-sheet-rich species according to the fluorescence intensity at the plateau. The oligomerization kinetics of hIAPP from batch B was intermediate compared with those from the two others, but the fluorescence was 8 times less intense than that obtained from batch A2. This feature could explain the lower proportion of monomeric hIAPP in batch B already highlighted by CE. Thus, according to Table 3, $\beta$-sheet-rich fibrils were not formed neither with the same kinetics nor with the same proportions and/or morphologies for the three peptide batches. It seems that the lower purity of lots $\mathrm{B}$ and $\mathrm{C}$ leads to a decrease of the $\beta$-sheet-rich species production, as well as a certain delay in their formation. This result clearly demonstrates that the peptide source has a strong impact on the aggregation process of hIAPP. Peptide quality can greatly impact its tendency to get aggregated, and some lots may contain already a non-negligible proportion of early aggregated forms affecting dramatically the overall process. Typically, the presence of only peak 3 in $\mathrm{CE}$ associated to a large increase of ThT fluorescence in spectrofluorimetry are relevant markers to select a good peptide batch for conducting in vitro aggregation monitoring.

The CE-UV method used previously to analyze the purity of the peptide samples is also suitable to monitor in real time the early in vitro oligomerization process of hIAPP [31]. This method was therefore used to compare the behavior of the three peptides regarding their aggregation. $\mathrm{CE}$ experiments were performed over $20 \mathrm{~h}$ of incubation in $50 \mathrm{mM}$ ammonium acetate buffer at $\mathrm{pH} 3.7$ (Fig. 5). As detailed previously, peaks 1 and 2 (or 2') are characteristic of oligomeric species, whereas peak 3 corresponds rather to hIAPP monomer. Peak 1 and 2 $\left(2^{\prime}\right.$ for batch B) areas all increased over time whatever the batch. However, the evolution of peak 3 overtime was markedly different. For batch A2, its area decreased to almost zero after $20 \mathrm{~h}$ of incubation. On the contrary, it remained constant for batch C. Its area decreased to zero for batch B but starting from a very low intensity as previously stated.

In batches $\mathrm{A} 2$ and $\mathrm{C}$, peaks 1 and 2 did not increase in the same proportion. They were more intense after $20 \mathrm{~h}$ of incubation for batch $\mathrm{C}$, indicating that this batch is producing a higher quantity of large oligomers. In addition, the monomer was much more maintained over time compared with what was observed with batch A2. For batch B, since the mobility of peak $2^{\prime}$ was different from that of peak 2 , we can assume that this peptide led overtime to the formation of alternative large oligomers different from those observed with batches A and $\mathrm{C}$.

\section{Concluding remarks}

Monitoring in vitro the hIAPP oligomerization is a key step to evaluate and identify new drug molecules targeting hIAPP oligomeric species. However, it remains very challenging due to the high variability observed during oligomerization experiments. We demonstrated that besides the techniques employed and the incubation medium selected, the peptide source is of paramount importance in the in vitro oligomerization assays. Considering that reproducibility of the different techniques used in that study was checked, differences observed are mainly attributable to the peptide source. Whereas similar results were observed for two lots from the same supplier, marked differences appeared with hIAPP provided by three different suppliers. The three techniques used, which give insights on $\beta$-sheet formation (ThT spectrofluorimetry), on chemical purity and conformational states (ESI-QToFIMS) and on monomer/large oligomer kinetics (CE-UV), provided different results for each supplier's peptide. The initial state of the peptide dramatically varied and consequently the lag phase observed by ThT fluorescence varied by a 3 -fold factor, while CE showed, in particular, a major difference in the kinetics of disappearance of the monomer peak. These results clearly demonstrate the paramount importance of the hIAPP peptide provenance in order to get reliable, reproducible, and comparable results on this aggregation process (both the early oligomerization and the latter fibrillization) from its monomer state. This must be particularly taken in consideration for the evaluation of the activity of molecules as anti- 
hIAPP oligomerization and fibrillization, in order to identify potential new drug candidates for the treatment of type 2 diabetes.

Funding information This work was supported by a public grant overseen by the French National Research Agency (ANR) as part of the "Investissement d'Avenir" program, through the "IDI 2015" project funded by the IDEX Paris-Saclay, ANR-11-IDEX-0003-02. This work was also supported by the DIM analytics from the Région Ile de France for MOBICS project funding.

\section{Compliance with ethical standards}

Conflict of interest The authors declare that they have no conflict of interest.

\section{References}

1. WHO (2019) Diabetes. https://www.who.int/health-topics/diabetes. Accessed 6 Oct 2019.

2. Pillay K, Govender P. Amylin uncovered: a review on the polypeptide responsible for type II diabetes. Biomed Res Int. 2013;2013: 826706. https://doi.org/10.1155/2013/826706.

3. Higham CE, Hull RL, Lawrie L, Shennan KI, Morris JF, Birch NP, et al. Processing of synthetic pro-islet amyloid polypeptide (proIAPP) "amylin" by recombinant prohormone convertase enzymes, PC2 and PC3, in vitro. Eur J Biochem. 2000;267:49985004.

4. Haataja L, Gurlo T, Huang CJ, Butler PC. Islet amyloid in type 2 diabetes, and the toxic oligomer hypothesis. Endocr Rev. 2008;29: 303-16. https://doi.org/10.1210/er.2007-0037.

5. Jeong HR, An SS. Causative factors for formation of toxic islet amyloid polypeptide oligomer in type 2 diabetes mellitus. Clin Interv Aging. 2015;10:1873-9. https://doi.org/10.2147/CIA. S95297.

6. Nguyen PT, Andraka N, De Carufel CA, Bourgault S. Mechanistic contributions of biological cofactors in islet amyloid polypeptide amyloidogenesis. J Diabetes Res. 2015;2015:515307. https://doi. org $/ 10.1155 / 2015 / 515307$.

7. Bedrood S, Li Y, Isas JM, Hegde BG, Baxa U, Haworth IS, et al. Fibril structure of human islet amyloid polypeptide. J Biol Chem. 2012;287:5235-41. https://doi.org/10.1074/jbc.M111.327817.

8. Hoffmann A, Caillon L, Salazar Vazquez LS, Spath P-A, Carlier L, Khemtémourian L, et al. Time dependence of NMR observables reveals salient differences in the accumulation of early aggregated species between human islet amyloid polypeptide and amyloid- $\beta$. Phys Chem Chem Phys. 2018. https://doi.org/10.1039/ C7CP07516B.

9. Abedini A, Plesner A, Cao P, Ridgway Z, Zhang J, Tu LH, et al. Time-resolved studies define the nature of toxic IAPP intermediates, providing insight for anti-amyloidosis therapeutics. Elife. 2016;5:e12977. https://doi.org/10.7554/eLife.12977.

10. Chen C-H, Yao T, Zhang Q, He Y-M, Xu L-H, Zheng M, et al. Influence of trehalose on human islet amyloid polypeptide fibrillation and aggregation. RSC Adv. 2016;6:15240-6. https://oi.org/ 10.1039/C5RA27689F.

11. Azriel R, Gazit E. Analysis of the minimal amyloid-forming fragment of the islet amyloid polypeptide. An experimental support for the key role of the phenylalanine residue in amyloid formation. $\mathrm{J}$ Biol Chem. 2001;276:34156-61. https://doi.org/10.1074/jbc. M102883200.
12. Khurana R, Coleman C, Ionescu-Zanetti C, Carter SA, Krishna V, Grover RK, et al. Mechanism of thioflavin $\mathrm{T}$ binding to amyloid fibrils. J Struct Biol. 2005;151:229-38. https://doi.org/10.1016/j. jsb.2005.06.006.

13. Pilkington EH, Xing Y, Wang B, Kakinen A, Wang M, Davis TP, et al. Effects of protein Corona on IAPP amyloid aggregation, fibril remodelling, and cytotoxicity. Sci Rep. 2017;7:2455. https://doi. org/10.1038/s41598-017-02597-0.

14. Sebastiao M, Quittot N, Bourgault S. Thioflavin T fluorescence to analyse amyloid formation kinetics: measurement frequency as a factor explaining irreproducibility. Anal Biochem. 2017;532:83-6. https://doi.org/10.1016/j.ab.2017.06.007.

15. Young LM, Cao P, Raleigh DP, Ashcroft AE, Radford SE. Ion mobility spectrometry-mass spectrometry defines the oligomeric intermediates in amylin amyloid formation and the mode of action of inhibitors. J Am Chem Soc. 2014;136:660-70. https://doi.org/ 10.1021/ja406831n.

16. Fortin JS, Benoit-Biancamano M-O. Inhibition of islet amyloid polypeptide aggregation and associated cytotoxicity by nonsteroidal anti-inflammatory drugs. Can J Physiol Pharmacol. 2016;94: 35-48. https://doi.org/10.1139/cjpp-2015-0117.

17. Gong H, Zhang X, Cheng B, Sun Y, Li C, Li T, et al. Bisphenol A accelerates toxic amyloid formation of human islet amyloid polypeptide: a possible link between bisphenol A exposure and type 2 diabetes. PLoS One. 2013;8:e54198. https://doi.org/10.1371/ journal.pone.0054198.

18. Bram Y, Frydman-Marom A, Yanai I, Gilead S, Shaltiel-Karyo R, Amdursky N, et al. Apoptosis induced by islet amyloid polypeptide soluble oligomers is neutralized by diabetes-associated specific antibodies. Sci Rep. 2014;4: SICI 4267. https://doi.org/10.1038/ srep04267.

19. Riba I, Barran PE, Cooper GJS, Unwin RD. On the structure of the copper-amylin complex. Int J Mass Spectrom. 2015;391:47-53. https://doi.org/10.1016/j.ijms.2015.09.001.

20. Cheng B, Gong H, Li X, Sun Y, Zhang X, Chen H, et al. Silibinin inhibits the toxic aggregation of human islet amyloid polypeptide. Biochem Biophys Res Commun. 2012;419:495-9. https://doi.org/ 10.1016/J.BBRC.2012.02.042.

21. Patel HR, Pithadia AS, Brender JR, Fierke CA, Ramamoorthy A. In search of aggregation pathways of IAPP and other amyloidogenic proteins: finding answers through NMR spectroscopy. J Phys Chem Lett. 2014;5:1864-70. https://doi.org/10.1021/jz5001775.

22. Hoffmann A, Saravanan MS, Lequin O, Killian JA, Khemtemourian L. A single mutation on the human amyloid polypeptide modulates fibril growth and affects the mechanism of amyloid-induced membrane damage. Biochim Biophys Acta Biomembr. 2018. https://doi.org/10.1016/J.BBAMEM.2018.02. 018.

23. Li H, Ha E, Donaldson RP, Jeremic AM, Vertes A. Rapid assessment of human amylin aggregation and its inhibition by copper(II) ions by laser ablation electrospray ionization mass spectrometry with ion mobility separation. Anal Chem. 2015;87:9829-37. https://doi.org/10.1021/acs.analchem.5b02217.

24. Young LM, Saunders JC, Mahood RA, Revill CH, Foster RJ, Tu LH, et al. Screening and classifying small-molecule inhibitors of amyloid formation using ion mobility spectrometry-mass spectrometry. Nat Chem. 2015;7:73-81. https://doi.org/10.1038/nchem. 2129.

25. Young LM, Saunders JC, Mahood RA, Revill CH, Foster RJ, Ashcroft AE, et al. ESI-IMS-MS: a method for rapid analysis of protein aggregation and its inhibition by small molecules. Methods. 2016;95:62-9. https://doi.org/10.1016/j.ymeth.2015.05.017.

26. Dupuis NF, Wu C, Shea JE, Bowers MT. The amyloid formation mechanism in human IAPP: dimers have beta-strand monomermonomer interfaces. J Am Chem Soc. 2011;133:7240-3. https:// doi.org/10.1021/ja1081537. 
27. Marek PJ, Patsalo V, Green DF, Raleigh DP. Ionic strength effects on amyloid formation by amylin are a complicated interplay among Debye screening, ion selectivity, and Hofmeister effects. Biochemistry. 2012;51:8478-90. https://doi.org/10.1021/ bi300574r.

28. Suzuki Y, Brender JR, Hartman K, Ramamoorthy A, Marsh EN. Alternative pathways of human islet amyloid polypeptide aggregation distinguished by (19)f nuclear magnetic resonance-detected kinetics of monomer consumption. Biochemistry. 2012;51:8154 62. https://doi.org/10.1021/bi3012548.

29. Suvorina MY, Selivanova OM, Grigorashvili EI, Nikulin AD, Marchenkov VV, Surin AK, et al. Studies of polymorphism of amyloid- $\beta 42$ peptide from different suppliers. J Alzheimers Dis. 2015;47:583-93. https://doi.org/10.3233/JAD-150147.

30. Verpillot R, Otto M, Klafki H, Taverna M. Simultaneous analysis by capillary electrophoresis of five amyloid peptides as potential biomarkers of Alzheimer's disease. J Chromatogr A. 2008;1214: 157-64. https://doi.org/10.1016/j.chroma.2008.10.051.

31. Berardet C, Kaffy J, Ongeri S, Taverna M. A capillary electrophoresis method to investigate the oligomerization of the human islet amyloid polypeptide involved in type 2 diabetes. J Chromatogr A. 2018. https://doi.org/10.1016/j.chroma.2018.10.006.

32. Richardson K, Langridge D, Giles K. Fundamentals of travelling wave ion mobility revisited: I. Smoothly moving waves. Int J Mass Spectrom. 2018;428:71-80. https://doi.org/10.1016/j.ijms.2018.03. 007.

33. Van der Rest G, Rezaei H, Halgand F. Monitoring conformational landscape of ovine prion protein monomer using ion mobility coupled to mass spectrometry. J Am Soc Mass Spectrom. 2017;28:303-14. https://doi.org/10.1007/s13361-016-1522-x.

34. Van der Rest G, Halgand F. Size exclusion chromatography-ion mobility-mass spectrometry coupling: a step toward structural biology. J Am Soc Mass Spectrom. 2017;28:2519-22. https://doi.org/ 10.1007/s13361-017-1810-0.

Publisher's note Springer Nature remains neutral with regard to jurisdictional claims in published maps and institutional affiliations. 